Notfall Rettungsmed 2009 • 12:339-340

DOI 10.1007/s10049-009-1179-0

Online publiziert: 30 Juli 2009

(c) Springer Medizin Verlag 2009

\author{
C. Waydhas ${ }^{1} \cdot$ C.K. Lackner ${ }^{2}$ \\ ${ }^{1}$ Klinik für Unfallchirurgie, Universitätsklinikum Essen \\ ${ }^{2}$ Institut für Notfallmedizin und Medizinmanagement (INM), \\ Klinikum der Universität München
}

\title{
Ausbildung und Lehre in der Notfallmedizin
}

Auf den ersten Blick erscheint das Thema Ausbildung in der Notfallmedizin in den Augen vieler aktiv tätiger Notärzte für die konkrete medizinische Versorgung von akut verletzten oder erkrankten Personen nicht von großer Relevanz.

Bei genauerem Hinsehen reicht es allerdings tief in das Selbstverständnis nicht nur der Notarzttätigkeit sondern der gesamten Rettungskette hinein. Der Notarzt mag noch so qualifiziert sein, der Behandlungserfolg hängt wesentlich von einer sofortigen korrekten Erste-Hilfe-Behandlung durch Laien, einer möglichst frühen Alarmierung des professionellen Rettungsdienstes und dem reibungslosen Funktionieren des Rettungsteams (Rettungsassistenten und Notarzt) ab. Auch die situative/individuelle Handlungskompetenz des Notarztes unterliegt großen Schwankungen. Die Vermittlung von Kenntnissen und ein zielgerichtetes praxisorientiertes Training, das evidenzbasiert ist und den Erfordernissen der präklinischen Notfallmedizin entspricht, sind für Medizinstudierende und angehende Notärzte gleichermaßen zwingend erforderlich.

Verschiedene Studien haben den Nutzen von öffentlich zugänglichen automatisierten Defibrillatoren („public access defibrillation") in den USA oder im Bereich der Luftfahrt gut belegt. Der Artikel von Urban et al. [3] stellt die Übertragbarkeit dieser Erkenntnisse auf das deutsche Rettungssystem dar und gibt einen Überblick, wie der medizinische Nutzen solcher Programme hier zu Lande ist, wo die Aufstellung von automatisierten externen Defibrillatoren besonders sinnvoll sein kann und auf welche Zielgruppen die Laienausbildung ausgerichtet sein soll.
Noch früher setzen Ausbildungsprogramme an, die Schüler in Erster Hilfe ausbilden. Hier kann der Grundstein dafür gelegt werden, dass Hilfsbereitschaft, Begeisterung und Idealismus vieler junger Menschen gefördert werden und dass sie als Erwachsene nicht nur bereit sind zu helfen sondern auch fachlich in der Lage dazu sind. Im Sinne einer Auffrischung schon vorhandener Kenntnisse wirken spätere weitere Schulungen in Erster Hilfe, wie etwa zum Erwerb des Führerscheins, vertiefend und nachhaltig.

\section{- Eine zielgerichtete Ausbildung schon der Medizinstudierenden und der angehenden Notärzte ist zwingend erforderlich.}

Die Basis notfallmedizinischer Kenntnisse wird den angehenden (Not-)Ärzten und Ärztinnen im Studium vermittelt. Die Umfrage und Analyse von Beckers et al. [1] zeigt dabei Gemeinsamkeiten, vielmehr aber noch die Variabilität der studentischen Ausbildung. Je nach Universität starten junge Mediziner mit ganz unterschiedlichen notfallmedizinischen Kenntnissen und Erfahrungen in die weitere Ausbildung, sollen aber den gleichen Anforderungen an die Behandlungsqualität gerecht werden. Der Artikel zeigt klar auf, dass die Schaffung eines einheitlichen Basis-Curriculums für die studentische Ausbildung in der Notfallmedizin ein zentraler Schritt zu einer vergleichbaren Ausbildungsqualität in ganz Deutschland ist.

Darauf aufbauend werden Mediziner durch eine klinische Tätigkeit mit in der Realität sehr heterogenem notfallmedizinischem Bezug und einen 8o Stunden umfassenden speziellen Kurs auf ihre Tätigkeit als Notarzt vorbereitet.

Die Verbesserung der Ausbildungsqualität von Notärzten darf aber hier nicht halt machen. Vielmehr erscheint es erstrebenswert, die wissenschaftlichen Erkenntnisse in der präklinischen Versorgung, evidenz- und konsensbasierte Leitlinien sowie etablierte und validierte Kurskonzepte in die von den Ärztekammern geforderte Weiterbildung zum Erwerb notfallmedizinischer Qualifikationen verstärkt und strukturiert einzubringen. Wege hierzu werden im Beitrag von Brokmann et al. [2] in eindrücklicher Weise aufgezeigt.

Nicht zuletzt hängt der Erfolg präklinischer Maßnahmen in starkem Maße vom Funktionieren der Rettungsteams ab. Eine reibungslose Zusammenarbeit von ärztlichem und nichtärztlichem Rettungsdienstpersonal ist dafür eine wichtige Voraussetzung. Dass dies, insbesondere unter stressbehafteten Situationen lebensbedrohlicher Störungen, nicht immer der Fall ist, wissen viele der im Rettungsdienst tätigen Personen. Die Notwendigkeit, ad hoc wichtige Entscheidungen auf der Basis von ungenügenden Informationen zu treffen, und die damit verbundene psychische Belastung können die Kommunikation innerhalb des Teams und die Qualität der Behandlung zum Nachteil des Patienten beeinflussen. Das gezielte Training nicht nur der einzelnen Personen, sondern des gesamten Teams mittels realitätsnaher Simulatoren kann besser als die meisten anderen Maßnahmen auf die tatsächliche Notfallsituation vorbereiten

Aufgrund der immanenten tiefgreifenden Strukturentwicklungen im klinischen 
und niedergelassenen Bereich ist der gesamte Themenkomplex sehr aktuell und wird nach unserer Einschätzung in $\mathrm{Zu}$ kunft nachhaltig von Bedeutung bleiben.

Wir möchten mit den ausgewählten Themen zur Ausbildung in der Notfallmedizin ein breites Spektrum von erfolgversprechenden Ansätzen zur Qualitätsentwicklung vor- und zur Diskussion stellen und dabei möglichst konkrete Anstöße geben und Strategieansätze aufzeigen, die eigene Handlungskompetenz und die des Rettungssystems, in welchem man tätig ist, qualitätsgestützt weiterzuentwickeln. Wir wünschen Ihnen in diesem Sinne viele Anregungen beim Lesen!

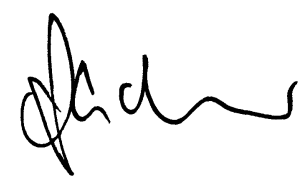

C.K. Lackner

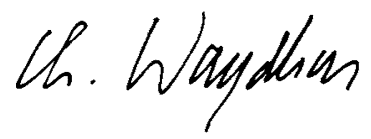

C. Waydhas

\section{Korrespondenzadresse}

\section{Prof. Dr. C. Waydhas}

Klinik für Unfallchirurgie,

Universitätsklinikum Essen

Hufelandstr. 55, 45147 Essen

christian.waydhas@uk-essen.de

\section{Literatur}

1. Beckers SK, Müller MP, Timmermann A et al (2009) Studentische Ausbildung in der Notfallmedizin. Überblick, Konzepte und Perspektiven. Notfall Rettungsmed 5. DOI 10.1007/s10049-009-1176-3

2. Brokmann JC, Beckers SK, Skorning M et al (2009) Evidenzbasierte Medizin in der notfallmedizinischen Fort- und Weiterbildung. Notfall Rettungsmed 5. DOI 10.1007/s10049-009-1178-1

3. Urban B, Hoffmann G, Ruppert M et al (2009) Laienausbildung. Hat „Public AccessDefibrillation" (PAD) eine Chance? Notfall Rettungsmed 5. DOI 10.1007/s10049-009-1174-5
„Notfall + Rettungsmedizin“ bietet Ihnen 8-mal im Jahr umfassende und aktuelle Beiträge zu interessanten Themenschwerpunkten (Leitthemen) aus allen Bereichen der Notfallmedizin.

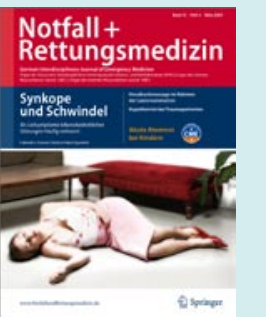

2008

- Heft 01/08 Evidenzbasierte Notfallmedizin

- Heft 02/08 ERC-Leitlinien 2005 - 2 Jahre danach

- Heft 03/08 Schlaganfall

- Heft 04/08 Luftrettung

- Heft 05/08 Invasive Notfalltechniken

- Heft 06/08 Polytrauma

- Heft 07/08 Postresuscitation Care

- Heft 08/08 Der psychiatrische Notfall

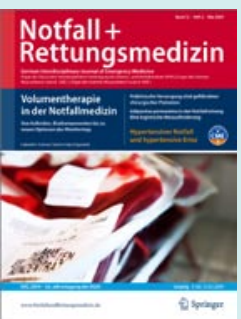

2009

- Heft 01/09 Die soziale Dimension der Notfallmedizin

- Heft 02/09 Synkope und Schwindel

- Heft 03/09 Volumentherapie in der Notfallmedizin

- Heft 04/09 Interdisziplinäre Notaufnahme

- Heft 05/09 Ausbildung in der Notfallmedizin

- Heft 06/09 Lebensbedrohliche Blutungen

- Heft 07/09 Rhythmusstörungen

- Heft 08/09 Kindernotfälle

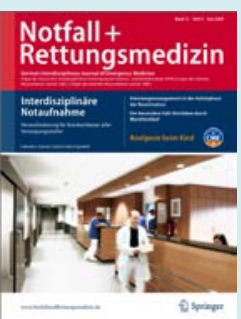

\section{Alle Beiträge auf einen Blick}

Abonnenten haben online Zugriff auf alle Beiträge im elektronischen Volltextarchiv unter

\section{www.NotfallundRettungsmedizin.de}

Ins Volltextarchiv gelangen Sie von der Startseite über den Navigationspunkt "Online-Archiv".

Eine interessante Lektüre wünscht Ihnen

Ihre Redaktion "Notfall + Rettungsmedizin" 